\title{
Virus Nanoblocks for Molecular Electronics
}

\author{
M.J. Kim,* A.S. Blum,** B. Gnade,* and B.R. Ratna** \\ *Department of Materials Science, University of North Texas, Denton, TX 76205 \\ **Center for Bio/Molecular Science and Engineering, Naval Research Lab., Washington DC
}

In the development of functional nanoscale devices, bottom-up approaches, where self-assembly and molecular recognition play a key role, are gaining more visibility [1]. Self-assembly is a basic building principle in nature for generating large arrays of biomolecules with well-defiened geometrical and physicochemical surface properties. The application of self-assembly for achieving perfect positional control at the molecular level appears feasible both theoretically and experimentally. For the fabrication of molecular electronic devices, one type of hierarchical selfassembly based on viruses is currently being studied. Use of a self-assembling plant virus particle (referred to as virus nanoblock or VNB) as a scaffold on which molecular electronic devices will be fabricated is a new approach and initial results are reported in this paper.

Figure 1(a) is a TEM image of icosahedral Cowpea Mosaic Viruses (CPMV), stained with Osmium tetroxide for imaging. CPMV has been chosen for this study because of the intense molecular, biological and structural information available on this group of viruses. The icosaheral shape of the CPMV capsid is built from 60 copies of asymmetric protein subunits. Its molecular model is shown in Fig. 1(b). The spherically averaged diameter of the capsid is $28.4 \mathrm{~nm}$. The observed size of the virus is very close to the predicted one, as shown in Fig. 1(a).

Specific functionalities of the virus capsid can be utilized to attach metal or oxide nanoparticles on the surface of the virus. Each virus particle has 12 5-fold, 203 -fold and 302 -fold symmetry axes due to its icosahedral symmetry. Because of various symmetry axes present on the virus surface, the particles will generate self-similar motifs [2]. For example, the SH end groups on the terminus of the cysteines can lead to attachment of gold nanoparticles. Depending on the site chosen and its location with respect to the 2, 3 and 5-fold symmetry axes a variety of decorations on the capsid can be envisaged. Figure 2(a) shows site specific attached gold nanoparticles on a virus. The observed stacking of gold nanoparticles is closely related to the model shown in Fig. 2(b). It is apparent that some of the gold nanoparticles were lost during the staining and TEM sample preparation processes. Most of the observed gold nanoparticles appear to be spherical in shape and their average size is about $5.5 \mathrm{~nm}$. Some of gold particles exhibited surface facets, as shown in Fig. 3. Our results clearly show self-assembling nature of the virus particle with site-specific attached gold nanoparticles. The feasibility of building nanocircuits and nanoscale devices from this bottom-up approach appears to be more realistic [3].

\section{Reference:}

[1] Breakthrough of the Year - Molecules Get Wired, Science 294 (2001) 2442.

[2] Q. Wang, T. Lin, L. Tang, J.E. Johnson and M.G. Finn, Angew. Chem. Int. Ed. 41 (2002) 459.

[3] This research was supported by DARPA Moletronics Program and UNT Faculty Research

Grant. The authors thank the Scripps Research Institute for the CPMV samples. 

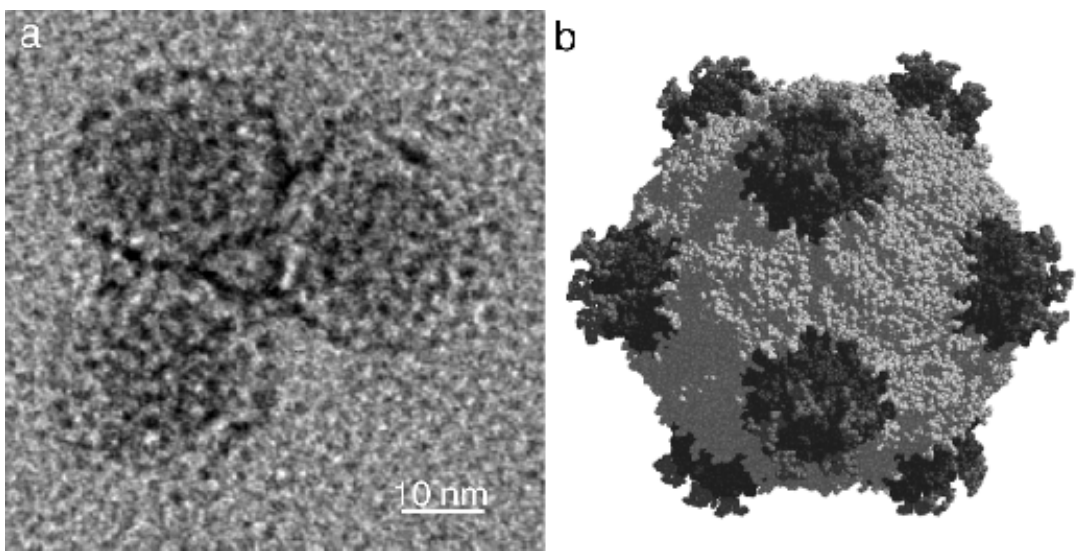

FIG. 1. (a) TEM image of the icosahedral viruses, stained with Osmium tetroxide. (b) The molecular model of a CPMV chimera showing a peptide insertion around the five-fold symmetry axes.
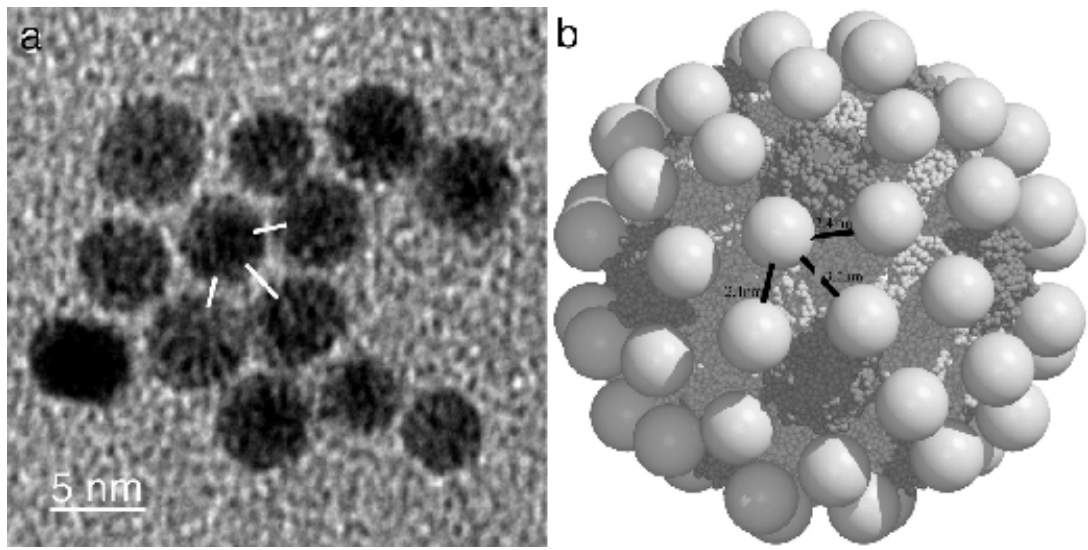

FIG. 2. (a) TEM image of gold nanoparticles attached to the virus. (b) Model showing a virus with site-specific attached gold nanoparticles. Some of the gold nanoparticle were lost during the staining process. Most of the observed gold nanoparticles appear to be spherical in shape and their average size is about $5.5 \mathrm{~nm}$.
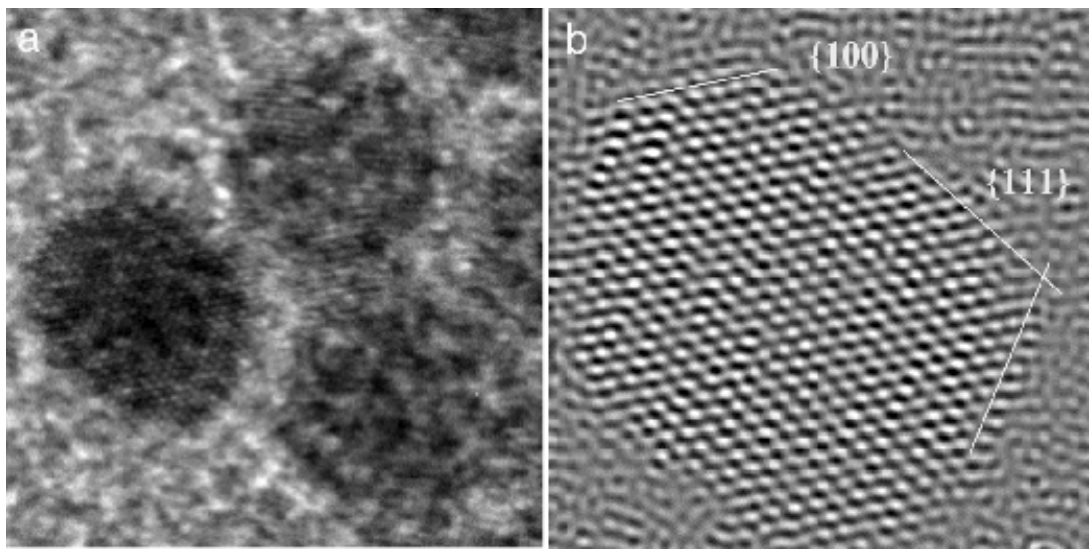

FIG. 3. (a) HREM image of gold nanoparticles, showing $\{111\}$ lattice fringes. (b) Fourier filtered image of a gold nanoparticle, showing surface facets. 